\title{
Multi-cohort analysis of colorectal cancer metagenome identified altered bacteria across populations and universal bacterial markers
}

Zhenwei Dai ${ }^{1,2 \dagger}$, Olabisi Oluwabukola Coker ${ }^{1,2 \dagger}$, Geicho Nakatsu ${ }^{1,2 \dagger}$, William K. K. Wu ${ }^{1,2+}$, Liuyang Zhao ${ }^{1,2}$, Zigui Chen ${ }^{3}$, Francis K. L. Chan ${ }^{1,2}$, Karsten Kristiansen ${ }^{4,5}$, Joseph J. Y. Sung ${ }^{1,2}$, Sunny Hei Wong ${ }^{1,2^{*}}$ (D) and Jun Yu ${ }^{1,2^{*}}$

\begin{abstract}
Background: Alterations of gut microbiota are associated with colorectal cancer (CRC) in different populations and several bacterial species were found to contribute to the tumorigenesis. The potential use of gut microbes as markers for early diagnosis has also been reported. However, cohort specific noises may distort the structure of microbial dysbiosis in CRC and lead to inconsistent results among studies. In this regard, our study targeted at exploring changes in gut microbiota that are universal across populations at species level.

Results: Based on the combined analysis of 526 metagenomic samples from Chinese, Austrian, American, and German and French cohorts, seven CRC-enriched bacteria (Bacteroides fragilis, Fusobacterium nucleatum, Porphyromonas asaccharolytica, Parvimonas micra, Prevotella intermedia, Alistipes finegoldii, and Thermanaerovibrio acidaminovorans) have been identified across populations. The seven enriched bacterial markers classified cases from controls with an area under the receiver-operating characteristics curve (AUC) of 0.80 across the different populations. Abundance correlation analysis demonstrated that CRC-enriched and CRC-depleted bacteria respectively formed their own mutualistic networks, in which the latter was disjointed in CRC. The CRC-enriched bacteria have been found to be correlated with lipopolysaccharide and energy biosynthetic pathways.

Conclusions: Our study identified potential diagnostic bacterial markers that are robust across populations, indicating their potential universal use for non-invasive CRC diagnosis. We also elucidated the ecological networks and functional capacities of CRC-associated microbiota.
\end{abstract}

Keywords: Colorectal cancer, Microbiota, Diagnostic marker, Ecology

\section{Background}

Colorectal cancer (CRC) is one of the most common cancers in the world with over 1 million cases diagnosed every year [1]. Many risk factors, including genetic, dietary, and other environmental factors contribute to CRC. The association of CRC with an altered gut microbiota has been studied in different populations, identifying bacteria such as Fusobacterium nucleatum and Bacteroides

\footnotetext{
* Correspondence: wonghei@cuhk.edu.hk; junyu@cuhk.edu.hk

${ }^{\dagger}$ Equal contributors

${ }^{1}$ Institute of Digestive Disease and Department of Medicine and Therapeutics, State Key Laboratory of Digestive Disease, Li Ka Shing Institute of Health Sciences, The Chinese University of Hong Kong, Shatin, Hong Kong Full list of author information is available at the end of the article
}

fragilis that are associated with tumorigenesis [2]. In this regard, $F$. nucleatum was found to modify the tumor immune microenvironment [3], while B. fragilis could produce DNA-damaging genotoxins in host cells [4]. Prevotella has been reported to be enriched in proximal colon cancer [5] and associated with interleukin (IL)-17producing cells [6]. Porphyromonas has also been identified to be associated with CRC in different populations [7-9]. The potential use of these microbes as non-invasive biomarkers for the detection of CRC has been explored $[10,11]$. However, studies from different populations may produce cohort-specific results. Furthermore, the gut microbiome is highly dynamic and influenced by dietary, 
xenobiotic, physiological, host genetics, and other factors [12], implying that results from metagenomic studies may not be applicable across different populations. Technically, metagenomic studies are also influenced by sample qualities, sequencing platforms, and the bioinformatic pipelines used for analysis [13]. These factors may result in heterogeneity and inconsistency among studies. Metaanalysis has an advantage of increasing statistical power over individual studies [12]. With a larger sample size, meta-analysis can pinpoint differences that are too small to be detected by single cohort studies while simultaneously considering population-specific characteristics [12]. By combining 16S rRNA gene sequence data sets from nine studies, Shah and colleagues recently identified a general composite microbial marker for CRC [14]. Nevertheless, interpretation of results generated by $16 \mathrm{~S}$ rRNA sequencing may be limited by its low taxonomical and functional resolution. The use of shotgun metagenomics sequencing allows the identification of bacterial taxa to species level [14] and is useful for analyzing gut microbiota functions without reliance on prediction [15]. We have previously reported on metagenomic features shared by CRC patients of different ethnicities [16]. We extended this work by performing a comprehensive meta-analysis of shotgun metagenomic data acquired from CRC patients and control subjects of American, Austrian, Chinese, and German and French cohorts to achieve greater statistical power to investigate the association of gut microbiota with CRC.

\section{Results}

Microbiota composition across cohorts

We accumulated shotgun metagenomic sequencing sequences from four cohorts (USA (USA), Austria (AT), China (HK), and Germany and France (FD)), including 271 controls and 255 CRC cases (demographic, clinical, and technical details are shown in Table 1). The sequences were curated, and we used Kraken v_0.10.5-beta for sequence classification and alignment. The Shannon diversity indexes were not significantly different between CRC cases and controls in USA, AT, and FD cohorts. Nevertheless, the diversity index decreased significantly in cases compared to controls in HK cohort $(p=0.045$, Additional file 1: Figure S1A). Principal coordinate analysis based on Bray-Curtis dissimilarity index identified significant bacteria compositional difference among cohorts (PERMANOVA, in control samples, $p<1 \times 10^{-4}$, Fig. 1a; in CRC samples, $p<1 \times 10^{-4}$, Fig. 1b). We also found significant differences in overall bacterial composition between cases and controls (PERMANOVA, $p<1 \times 10^{-4}$, Fig. 1c).

\section{Bacteria differing in abundance between CRC cases and controls across cohorts}

We performed simulation analysis to compare the statistical powers between the meta-analysis and single cohort studies. Our power simulation analysis showed an increased statistical power with the rank sum meta-analysis approach. Simulation analysis showed an estimated power

Table 1 Fecal samples' demographic, clinical, and technical details

\begin{tabular}{|c|c|c|c|c|c|c|}
\hline Cohort & Factor & Control & CRC & $P$ value & Sample collection & Sequencing platform \\
\hline \multirow[t]{4}{*}{ Cohort C1 (American, 2016) } & Sample size & 52 & 48 & NA & \multirow[t]{4}{*}{ Prior to surgery and treatment } & \multirow{16}{*}{$\begin{array}{l}\text { Sequencing Platform: Illumina } \\
\text { Hiseq 2000/2500; Sequencing } \\
\text { Target Depth: 5GB; read length } \\
100 \text { bp }\end{array}$} \\
\hline & Age & $61.23(11.03)$ & $60.96(13.56)$ & 0.913 & & \\
\hline & Gender & $\begin{array}{l}\text { Male:37; } \\
\text { Female:15 }\end{array}$ & $\begin{array}{l}\text { Male:35; } \\
\text { Female:13 }\end{array}$ & 1 & & \\
\hline & BMI & $25.35(4.27)$ & $24.90(4.29)$ & 0.601 & & \\
\hline \multirow[t]{4}{*}{ Cohort C2 (Austrian, 2014) } & Sample size & 63 & 46 & NA & \multirow[t]{4}{*}{ Not available } & \\
\hline & Age & $67.1(6.37)$ & $67.1(10.91)$ & 0.999 & & \\
\hline & Gender & $\begin{array}{l}\text { Male:37; } \\
\text { Female:26 }\end{array}$ & $\begin{array}{l}\text { Male:28; } \\
\text { Female:18 }\end{array}$ & 0.978 & & \\
\hline & BMI & $27.57(3.78)$ & $26.50(3.53)$ & 0.132 & & \\
\hline \multirow[t]{4}{*}{ Cohort C3 (Chinese, 2015) } & Sample size & 92 & 73 & NA & \multirow{4}{*}{$\begin{array}{l}\text { No antibiotics and no invasive } \\
\text { medical intervention for } 3 \text { months; } \\
\text { no vegetarian diet; no history of } \\
\text { cancer or inflammatory disease of } \\
\text { intestine }\end{array}$} & \\
\hline & Age & $58.51(7.55)$ & $65.90(10.61)$ & $<0.0001$ & & \\
\hline & Gender & $\begin{array}{l}\text { Male:51; } \\
\text { Female:41 }\end{array}$ & $\begin{array}{l}\text { Male:47; } \\
\text { Female:26 }\end{array}$ & 0.316 & & \\
\hline & BMI & $23.87(3.31)$ & $24.07(3.18)$ & 0.697 & & \\
\hline \multirow{4}{*}{$\begin{array}{l}\text { Cohort C4 (German and } \\
\text { French, 2014) }\end{array}$} & Sample size & 64 & 88 & NA & \multirow{4}{*}{$\begin{array}{l}\text { No previous colon or rectal } \\
\text { surgery, colorectal cancer, } \\
\text { inflammatory, or infectious } \\
\text { injuries of the intestine; no need } \\
\text { for need for emergency } \\
\text { colonoscopy }\end{array}$} & \\
\hline & Age & $58.75(12.96)$ & $68.44(12.22)$ & 0.007 & & \\
\hline & Gender & $\begin{array}{l}\text { Male:32; } \\
\text { Female:32 }\end{array}$ & $\begin{array}{l}\text { Male:53; } \\
\text { Female:35 }\end{array}$ & 0.276 & & \\
\hline & BMI & $24.72(3.19)$ & 25.89(4.29) & 0.056 & & \\
\hline
\end{tabular}



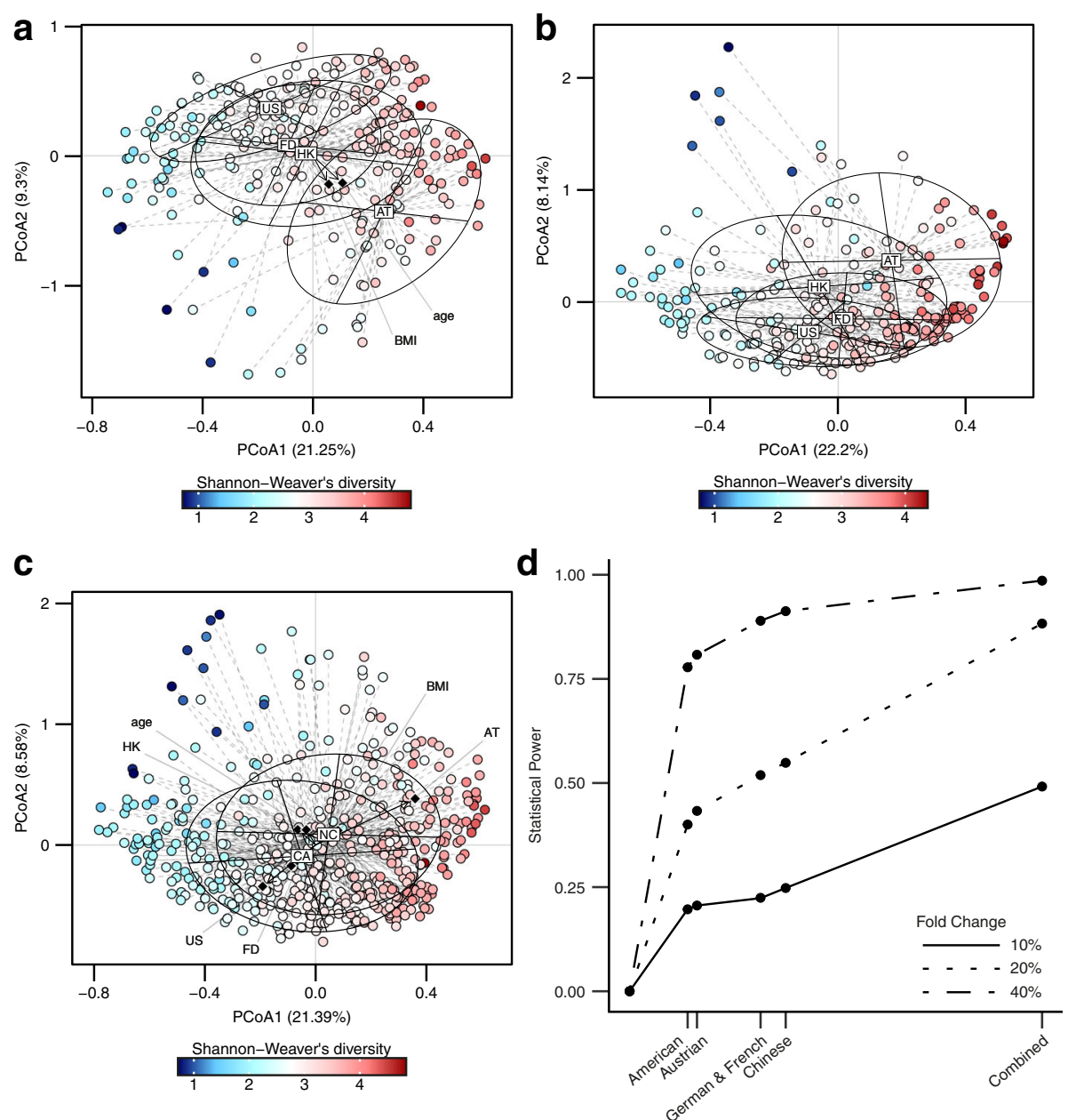

Fig. 1 Microbial composition and statistical power difference across cohorts. a-c Principal coordinate analysis for control samples, CRC samples, and all samples, respectively. (CA, CRC; NC, negative control) The correlations between phenotypes and PCoAs are labeled with their corresponding coordinates. d Statistical power to detect differentially abundant bacteria of various fold change (fold change $=10,20$, and $40 \%$ ) versus cohort sample size (number of control samples $\times$ number of case samples)

of 0.88 with a bacterial abundance change of $20 \%$. This shows an advantage over single-cohort studies with an approximate power of 0.5 at abundance fold change of $20 \%$ (Fig. 1d).

We applied this meta-analysis approach to identify bacterial species that exhibited differential abundance in CRC compared to controls across all the four cohorts. After excluding bacteria showing 'divergent directional changes', 994 species were obtained for further analysis (Additional file 2: Text). Using the rank sum method, we identified 7 enriched species and 62 depleted species in CRC cases compared to controls (Fig. 2a). We demonstrated that the significance levels (pfp) of the 69 different abundant species were not affected by our filtering pipeline (Additional file 3: Table S14). These results were validated with another independent pipeline, MetaPhlan [17] (Additional file 4: Table S4). The seven CRC-enriched species included Bacteroides fragilis, Fusobacterium nucleatum, Porphyromonas asaccharolytica,
Parvimonas micra, Prevotella intermedia, Alistipes finegoldii, and Thermanaerovibrio acidaminovorans.

Among the seven CRC-enriched species, Bacteroides fragilis was observed to be consistently enriched across all four cohorts (Fig. 2b), whereas five bacteria showed significant changes in abundance in two of the four cohorts ( $P$. asaccharolytica, P. micra, P. intermedia, A. finegoldii, and T. acidaminovorans). Five bacterial species among the 62 CRC depleted bacteria have been reported to confer health benefits, including Clostridium butyricum [18, 19], Streptococcus salivarius [20, 21], Streptococcus thermophilus [22], Carnobacterium maltaromaticum [23], and Lactobacillus gallinarum [24]. To identify whether the $69 \mathrm{CRC}$-associated bacteria correlated with CRC progression, we investigated their abundance difference between early- and late-stage CRC (Additional file 5: Table S1). Three species showed decreasing trends across control, early- and late-stage 


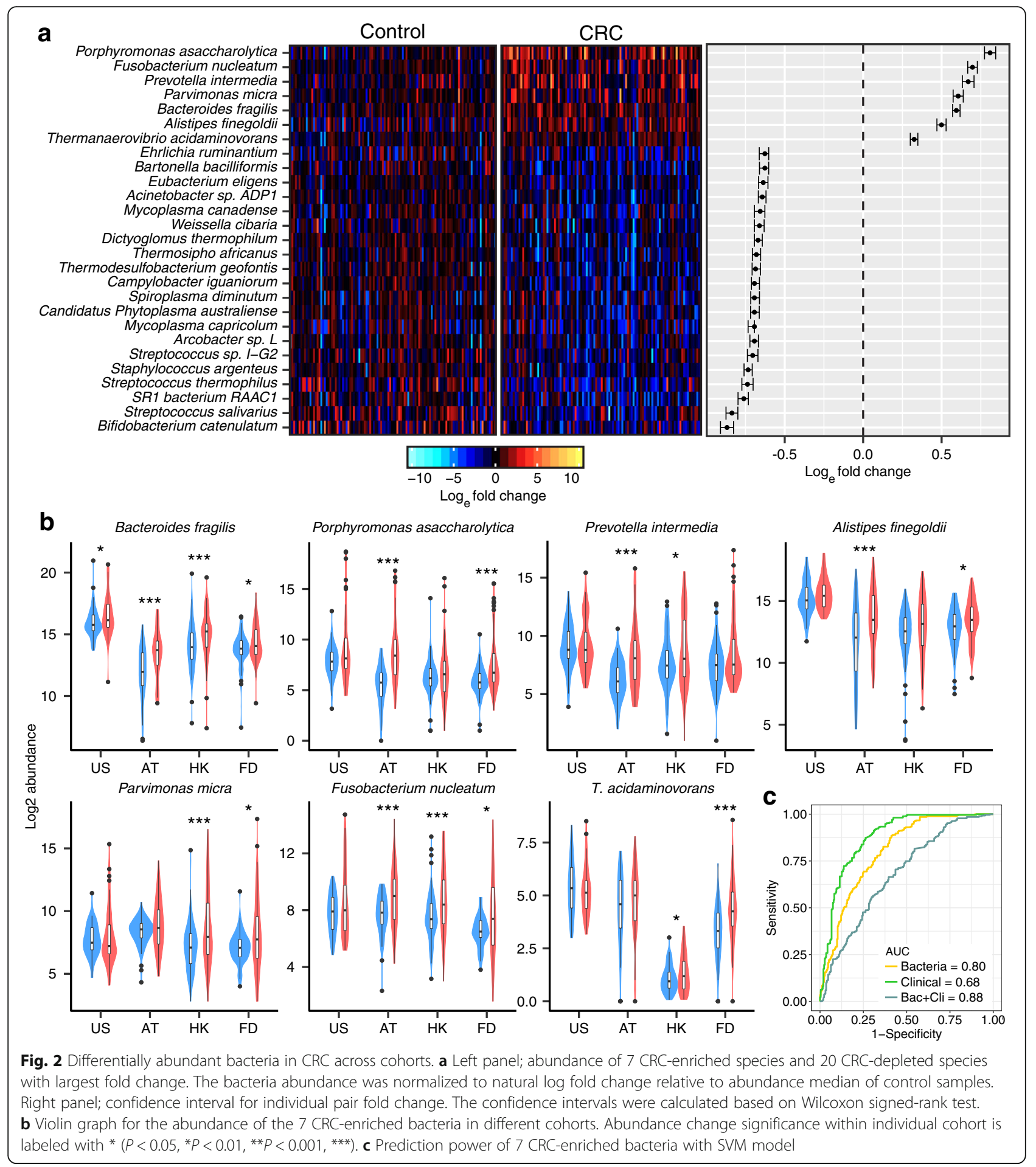

CRC (Streptococcus sp. I-G2, Shewanella woodyi, Mycoplasma penetrans, Additional file 6: Figure S5).

\section{Classification of CRC cases and controls with bacterial markers}

To classify CRC cases from controls based on bacterial composition, the seven CRC-enriched bacterial species were fitted into a support vector machine (SVM) model with radial kernel [25]. We obtained areas under the receiver-operating curve (AUCs) of $0.83,0.87,0.84$, and 0.82 , respectively, for USA, AT, HK, and FD cohorts (Additional file 7: Figure S3B). As we used a SVM model with 10-fold cross validation, it achieved average AUC of 0.75 on the testing fold (Additional file 8: Figure S12A). 
The overall AUC was 0.80 for the combined population, and this performance was not significantly skewed by a single cohort (Additional file 7: Figure S3A). We further evaluated the classification power of bacteria markers by leaving-one-cohort-out approach. We leaved one cohort as the validation samples at each time and used the three cohorts left to select markers and train the SVM model. Our model achieved an average AUC at 0.73 on the validation samples (Additional file 8: Figure S12B). After the inclusion of clinical phenotype information, namely, age, gender, and body mass index (BMI), the overall AUC increased to 0.88 (Fig. 2c). Optimal F1 scores (harmonic mean of recall and precision) could reach 0.67 and 0.80 , respectively using bacteria markers alone and combining clinical phenotype information (Additional file 9: Figure S4A, Additional file 10: Table S13 and Additional file 11: Table S15). Additionally, the potential of using the seven bacterial markers for diagnosis of early-stage CRC was evaluated. We calculated the significance of available bacterial abundance changes in AT, HK, and FD cohorts (cancer stage information is missing in USA). The results showed significant abundance changes for the seven CRCenriched species in three cohorts, indicating that data from the three cohorts (besides cohort USA) were sufficiently informative for the stage-specific analysis (Additional file 12: Figure S6A). Using the SVM model, the seven CRC-enriched species classified early-stage CRC patients from controls with AUCs of 0.84, 0.82, 0.84 in AT, HK, and FD cohorts, respectively (Additional file 12:
Figure S6B), suggesting an outstanding classification performance between early-stage CRC cases and controls.

\section{Correlations between CRC-related bacterial species}

To gain insights into the bacteria-bacteria interactions from an ecological perspective, we further investigated the correlations between the CRC-enriched and CRCdepleted bacteria based on SparCC algorithm. The average correlation strength across the populations was also estimated (see "Methods"). We observed that the enriched and depleted bacteria, respectively, formed their own mutualistic networks that were negatively correlated with each other (Additional file 13: Figure S7). Interestingly, the number of significant correlation pairs and correlation strengths among CRC-depleted bacteria were higher in controls than in CRC cases (difference of significant correlation proportions: $p=6.4 \times$ $10^{-5}$; difference of correlation strength: $p=2.6 \times 10^{-8}$ ) (Fig. 3b). Most of the correlations between CRC-enriched and CRC-depleted bacteria were negative. The seven CRC-enriched bacteria were more closely correlated in early-stage than late-stage $\mathrm{CRC}$, while the correlation networks between them was disrupted in late-stage CRC (Additional file 14: Figure S9C, correlation strength: $p=0.013$ ). With weighted degree centrality, we found that Clostridium species (Additional file 15: Table S2) had the highest centralities in the network. These central species may play a pivotal role in the network, supported by analyses of the global efficiency and weighted

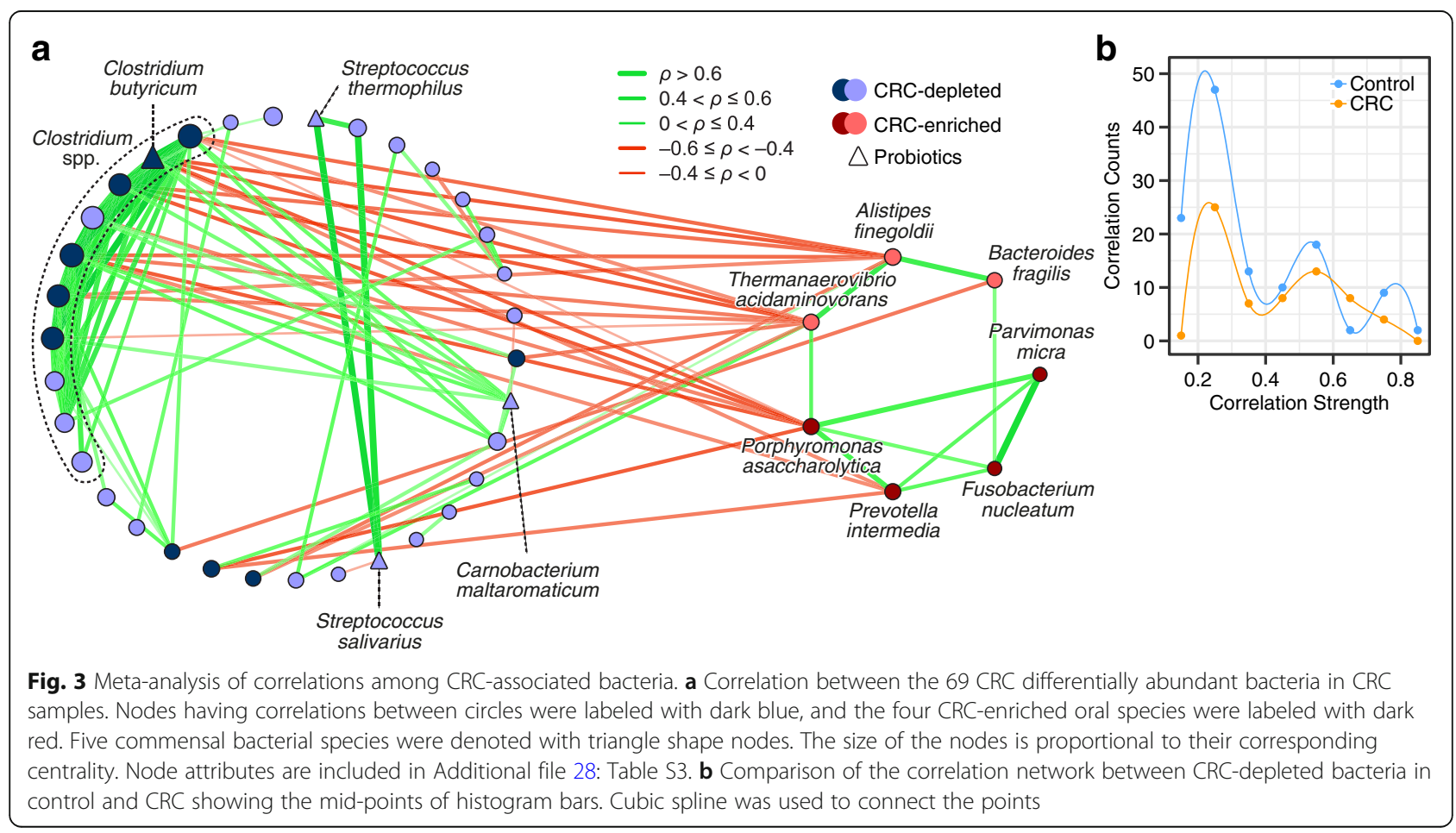


network connectance (Additional file 16: Figure S8). Compared with the removal of random nodes, the network connectivity decreased very sharply when nodes of Clostridium species were removed.

\section{Functional gene families associated with CRC-enriched and CRC-depleted bacteria}

The metagenome sequences were mapped to UniRef database and grouped into 10,675 gene ontology (GO) and 8695 KEGG ontology (KO) categories with HUMAnN2. The $\mathrm{GO} / \mathrm{KO}$ reads were normalized to relative abundance (copy per million units) for comparison. A total of 311 GO categories and $217 \mathrm{KO}$ categories were identified to be enriched in CRC $(\mathrm{FDR}<0.05)$; whereas $31 \mathrm{GO}$ categories and $74 \mathrm{KO}$ categories were depleted in CRC (Additional file 17: Table S6 and Additional file 18: Table S7). We investigated the correlation between the seven CRC-enriched species and $\mathrm{GO} / \mathrm{KO}$ categories with Spearman's correlation, and identified 167 GO categories and $143 \mathrm{KO}$ categories that have significant positive correlations $(\mathrm{FDR}<0.05)$ with the $\mathrm{CRC}$-enriched bacteria (Fig. 4, Additional file 16: Figure S8 and Additional file 14: Figure S9). We defined these GO/ $\mathrm{KO}$ categories as CRC-enriched bacteria correlated $\mathrm{GO} / \mathrm{KO}$ categories.

To investigate the functional pathways involved, we mapped the $\mathrm{KO}$ categories to the KEGG pathway. KO categories that were involved in the same pathway were treated as correlated $\mathrm{KO}$ categories. We observed 45 KEGG pathways involving at least two CRC enrichedbacteria correlated $\mathrm{KO}$ categories (Additional file 19: Table S10). To identify the pathways shared by multiple $\mathrm{KO}$ categories in association with cancer development, we found seven KEGG pathways whose overall

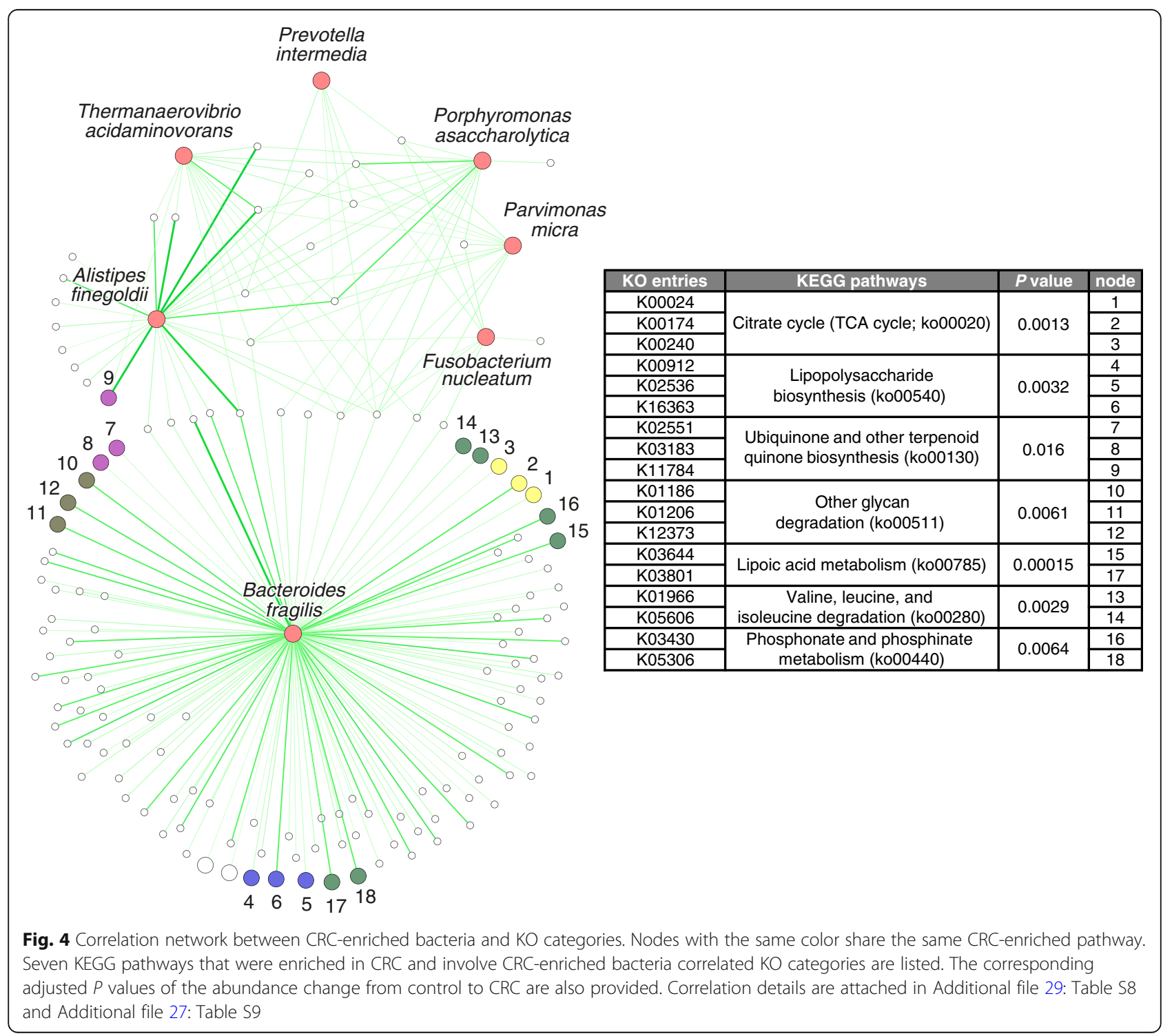


abundance were enriched in CRC, namely, ko00020 (Citrate cycle (TCA cycle)), ko00540 (Lipopolysaccharide biosynthesis), ko00130 (ubiquinone and other terpenoidquinone biosynthesis), ko00785 (lipoic acid metabolism), ko00280 (valine, leucine, and isoleucine degradation), ko00440 (phosphonate and phosphinatemetabolism), and ko00511 (other glycan degradation) (Fig. 4). Some commensal bacterial species were found to be correlated with CRC-depleted GO/KO categories; Clostridium butyricum and Carnobacterium maltaromaticum were strongly correlated with GO0051606 (stimulus detection; $\rho=0.56$ and 0.51, respectively) while Streptococcus salivarius and $S$. thermophiles were correlated with K07104 (catechol 2,3dioxygenase; $\rho=0.78$ and 0.67 , respectively) and K07570 (general stress protein $13 ; \rho=0.74$ and 0.66 , respectively) (Additional file 20: Figure S11).

\section{Discussion}

In this study, we performed the first comprehensive meta-analysis of shotgun metagenomics on CRC. We assembled samples from three different continents and four different ethnic cohorts including a large number of CRC cases and control fecal samples. We identified species-level bacterial markers that were enriched and depleted in CRC across cohorts with a robust statistical method, rank sum, which was a model-free approach suitable for handling non-normal data. Further analysis provided inferences about the correlations between the bacterial markers and their possible functional roles. This study shows how meta-analysis of shotgun metagenomics data can provide useful biological information by identifying biomarkers with higher statistical power.

Heterogeneity of the microbiota was observed across different cohorts. From the principal component analysis results, we observed very significant $P$ values for the difference in microbial composition among the populations. This observation is consistent with previous studies showing effects of ethnicity and technical differences on gut microbiota [26-28] and highlights the need for combined analysis.

The meta-analysis approach has been used to evaluate and combine results of comparable studies [29] with major advantages of increasing statistical power and improving estimates of effect size in association studies [30]. Our meta-analysis, using the rank sum method, identified seven bacterial markers that were CRCenriched across four cohorts. Our results are supported by the fact that six out of the seven identified CRCenriched bacteria, namely, $P$. asaccharolytica, $F$. nucleatum, B. fragilis, P. intermedia, P. micra, and A. finegoldii have been reported previously to be associated with CRC [5] in some but not all populations. P. micra was found to be significantly enriched in CRC and strongly correlated with F. nucleatum in Chinese cohort [16].
Our results suggest that $P$. micra can be universally associated with CRC across the cohorts. The Alistipes genus was found to be associated with CRC in a previous study [31], while $A$. finegoldii was isolated from the blood culture of CRC patient [32]. These observations support their roles in colorectal carcinogenesis. Prevotella was found to be enriched in proximal colon cancer [5], despite the controversial results reported in another study [33]. From our analysis, P. intermedia was clearly an enriched species in CRC, after combining the information from four cohorts. Interestingly, among the seven CRC-enriched species, four are oral bacteria $(P$. asaccharolytica, F. nucleatum, $P$. intermedia, and $P$. micra). Though the correlation between oral bacteria and CRC has been reported [34], our results suggest such a relationship exists across populations.

Our results also show a good performance of the seven CRC-enriched bacteria in classifying CRC from controls across cohorts. Population-specific variations may limit the classification performance of individual bacterial markers. For example, F. nucleatum has been used for CRC diagnosis in our previous study $(\mathrm{AUC}=0.85)[10]$, but it is unknown whether it may be discriminative for other populations (Fig. 2b). However, through metaanalysis, we found a set of bacterial markers that was robust to population variations, which was exemplified by the performance of the seven bacterial markers achieving AUCs of at least 0.80 across all four populations. Our study also has the advantage of an overall balanced case-control sample size, which, under similar AUCs, usually provides a higher $F_{1}$ score compared with a cohort with imbalanced case-control sample size due to accuracy paradox. As shown in our simulation analysis, when the sample size was imbalanced with a much smaller size in CRC cases than controls, the optimal $F_{1}$ score was much smaller. Our result suggests that the prudent addition of markers may improve diagnostic performance and emphasize the advantage of meta-analysis in identifying markers applicable to multiple populations.

Besides the CRC-enriched bacteria, we identified five bacterial species previously reported to confer health benefits among the 62 CRC-depleted bacteria. The protective role of the bacteria during the colorectal carcinogenesis has not been thoroughly studied. Clostridium butyricum, identified in this study, was previously found to promote the apoptosis of CRC cells and inhibit intestinal tumorigenesis in mice [35]. This supports our finding of negative association between these bacteria and CRC (Fig. 3), and suggest that they may potentially act as probiotics to inhibit CRC progression.

In addition to bacterial abundance, changes in bacterial correlations could partially explain colorectal tumorigenesis. A closely correlated network between the CRCdepleted bacteria which may play a role in stabilizing the 
gut microbiota was observed. Its disruption may potentially contribute to colorectal carcinogenesis. On the other hand, the negative correlations between CRC-enriched and -depleted bacteria suggest the possibility of reciprocally antagonistic effects between them. The depletion of some commensal bacteria could reduce the suppressive effects on CRC-enriched bacteria, contributing to their enrichment. We also observed some interesting changes of the bacterial correlation pairs. Two oral bacteria, P. micra and F. nucleatum, were proposed to be strongly correlated in CRC in a previous study [16], which was also validated in our meta-analysis. This correlation was observed not to be significant in control samples, indicating that the CRC environment may be an important factor for the formation of this correlation and the two species may function cooperatively in CRC.

The metabolic functions of microbiota associated with CRC remain largely unknown. Our analysis revealed some functional shifts related to bacterial enrichments. Bacterial changes in CRC may result in the alteration of some functional gene-families and pathways to contribute to colorectal carcinogenesis. According to our results, over $50 \%$ of the CRC-enriched $\mathrm{GO} / \mathrm{KO}$ categories significantly correlated with the CRC-enriched bacteria, suggesting their non-negligible contribution to the overall metabolic functionality in CRC. Interestingly, most of the $\mathrm{CRC}$-enriched $\mathrm{GO} / \mathrm{KO}$ categories that correlated with $B$. fragilis were discrete from other $\mathrm{CRC}$-enriched bacteria, suggesting that $B$. fragilis may function independently in CRC (Fig. 4, Additional file 21: Figure S10). We also found some CRC-associated pathways identified by previous researchers. This included the pathway related to lipopolysaccharide (LPS), a gram-negative bacterial antigen that can induce toll-like receptor 4 signaling and promote cell survival and proliferation in CRC [36]. LPS has been shown to enhance cell migration in esophageal cancer cell line [37]. Our observation of the enrichment of this pathway in CRC and its correlation with CRC-enriched bacteria suggests its role in CRC and supports previous observations. Other bacterial functions involving biosynthetic pathways, metabolism of cofactors and vitamins and energy production pathways positively correlated with the CRC-enriched bacteria. These pathways may serve as alternative bioenergetic sources for metabolically stressed cancer cells [38].

Though we considered possible confounding factors, namely age, gender and BMI, other potential confounders such as tumor location, comorbidities, and cancer status were not included in this study. We used a filtering pipeline to remove bacteria showing divergent abundance changes (Additional file 22: Figure S13). While this may have led to the missing of some interesting species, this approach should have minimized the false positive discovery rate. It is likely that the seven identified species are genuinely related to CRC. Statistically, the sparsity of the bacterial abundance data makes it difficult to select features accurately. Additionally, the correlation and functional analysis were performed based on bacterial abundance and sequencing data. The difference between the mucosal and fecal microbiota may lead to the inconsistency of our findings and the real changes of microbiota in gut during the colorectal carcinogenesis. Despite these limitations, our study aggregated and uniformly analyzed deep-sequenced microbial fecal samples from diverse populations and found bacterial species that were consistently enriched in CRC across cohorts. The shotgun metagenome sequences used in this study equipped us with species-level identification and allowed analyses on bacterial correlations and metabolic functions. Importantly, our study provides directions for further research on the CRC microbiota.

\section{Conclusions}

With the advancement in next generation sequencing technique, more metagenomic sequencing data sets are available, providing higher resolution of bacterial sequences. Though previous studies have widely reported the association between microbiota and CRC, it is essential to determine bacterial markers that are robust to population specific characteristics. Our study identified a group of bacteria that is consistently associated with CRC and shows potential in the diagnosis of CRC across multiple populations despite technical and biological variations. Universal ecological and functional shifts related to bacterial enrichment and depletion were also revealed, providing directions for further research on the potential functional involvements of the gut microbiota in contributing to colorectal tumorigenesis.

Future meta-analysis of gut microbiota dysbiosis in CRC should include more high quality metagenomic sequences especially from ethnic groups seldom covered by previous studies, such as African and south-east Asian, to provide higher statistical power and build up a more complete overview of CRC associated microbial dysbiosis. New methods for analyzing microbiome compositional data are also indispensable. Present analysis of the microbiome data treats every taxon independently, while the correlation structure between taxa has not been well incorporated, which may lead to high false discovery rate. With more available data sets and more powerful statistical analysis methods, better microbial CRC diagnostic tools and deeper understanding of microbial functions in colorectal carcinogenesis are obtainable.

\section{Methods}

Sequence curation and quality control

Raw sequences from CRC fecal metagenomics studies published from year 2014 to 2016 and with similar 
sequencing depths and target read lengths were retrieved from NCBI database [16, 31, 39, 40]. A total of 255 CRC patients and 271 controls from four cohorts (USA (US), Austria (AT), China (HK), and Germany \& France (FD)) were included in this meta-analysis. Whole-genome shotgun sequencing of the samples from all cohorts was carried out on Illumina HiSeq 2000/2500 (Illumina, San Diego, USA) platform with similar sequencing depths (read length $100 \mathrm{bp}$, target sequencing depth $5 \mathrm{~GB}$ ). Trimmomatic v_0.36 was used to remove low quality sequences. Human sequences were removed after alignment with reference genome (hg38 database [41]) using Bowtie2 v_2.2.9, with default settings. Kraken v_0.10.5beta was then used to for taxonomic classification of unmapped microbial reads [42]. By the default setting of Kraken, only complete genome sequences were included as the reference genome database to reduce the errors from contaminants [42]. Since our project focused on bacteria community, we only included the sequences whose lowest common ancestor can be aligned to bacteria database. In HK and FD cohorts, we removed one sample respectively with low bacterial read count compared with other samples (removed samples' bacteria read count $=57,547$ and 165,287 ). Species level read counts were rarefied to the minimum read counts in selected samples of each cohort, namely; 2,419,973, $1,596,424,1,222,507$ and 856,204 per sample, respectively, in US, AT, HK and FD cohorts to reduce the effects of uneven sampling in each cohort. Because the real sequencing depth varied across the cohorts, though their target sequencing depths were the same, we did not rarefy all the samples to the same read counts to maintain this difference. This approach gave us larger read counts in US, AT and HK cohorts, which could reduce the variance of the estimated relative abundance $\left(\operatorname{Var}\left(y_{i j} / y_{i+}\right)\right.$ decreases provided a larger $\left.y_{i+}\right)$. Principal coordinates analysis was used with Bray-Curtis dissimilarity matrix to visualize microbiota composition.

\section{Meta-analysis of differentially abundant bacteria}

We performed combined analyses on bacteria showing concordant changes in the individual study cohorts. We excluded a bacterial candidate from the analysis, if it exhibited significant contradictory abundance change directions among different cohorts. In our project, we gave a point estimation for the bacteria abundance change direction using the median. We removed species showing a balanced discrepancy in abundance changes in the four cohorts (positive abundance changes in two cohorts and negative abundance changes in two other cohorts), or species showing significantly discrepant change in one or more cohorts. Statistical significance was defined by $p<0.05$ by the Mann-Whitney $U$ test (see Additional file 2: Text for details). Considering the non-normality and over-dispersion of the microbial composition data, the non-parametric rank sum method was used for meta-analysis [43-45]. To control the confounding effects, any bacterial taxa whose abundance was significantly associated with the confounding variable was adjusted for the confounder using linear regression: $\log \left(y_{i}\right)=\beta_{0}+X_{i} * \beta_{1}, i=1,2, \ldots, n$; where $y_{i}$ is the abundance of sample $i$ and $X_{i}$ is the value of confounding variable of sample $i$. The adjusted bacteria abundance has the form $y_{i}=y_{i} * \exp \left(-X_{i} * \beta_{1}\right)$. With this approach, the effect of the confounder on the fold change was adjusted: $\frac{y_{i}^{\prime}}{y_{j}^{\prime}}=\frac{y_{i}}{y_{j}} * \exp \left(-\left(X_{i}-X_{j}\right) * \beta_{1}\right)$. Species with estimated percentage of false-positives (pfp) smaller than 0.01 were considered significant. The significance of bacterial abundance change in the individual cohort was estimated using conditioned Mann-Whitney test with COIN package in R. Age was observed to be a confounding factor in HK and FD cohorts (Table 1). Since age is continuous, we divided the ages into 4 groups (Additional file 23: Figure S2) and treated the age groups as the conditioned variable for the test.

\section{Evaluation of statistical power}

To evaluate the statistical power of rank sum metaanalysis method, we simulated microbial composition data following Dirichlet-multinomial distribution [46]. Most of the species were assumed to have similar relative abundance in CRC and control. Only a small proportion of the species (50 species) were simulated to be associated with CRC. We applied rank sum on the simulated microbial composition data and selected the top 50 species with the smallest rank sum statistics as bacteria exhibiting differential abundance. Among the 50 species, the proportion of real differentially abundant bacteria in the simulation data was used as an estimation of the statistical power. The statistical powers were compared under the fold change of $10 \%, 20 \%$, and $40 \%$.

\section{Correlation and network analyses}

Correlations between species were calculated with the SparCC algorithm, a robust method for correlation inference of sparse compositional data [47]. We calculated the correlation separately in the four cohorts. The overall correlation across the cohorts was estimated using Hedges and colleagues' method, where the correlations were first taken as Fisher's $r$-to- $Z$ transformation and then, calculated through weighted average of the transformed scores $[48,49]$. Only significant correlations with $P$ value $<0.05$ were included in the downstream analysis. Correlation estimation was implemented with package 'metacor' in R, and the network was visualized with Cytoscape v_3.4.0. The centrality of nodes in the correlation network was evaluated by weighted degree centrality 
using correlation strength as the weight. Network connectivity was measured by global efficiency and weighted network connectance, where the global efficiency is defined as $E_{\text {global }}(G)=\frac{E(G)}{E(G \text { ideal })}=\frac{2}{n(n-1)} * \sum_{i \neq j \in G} \frac{1}{d(i, j)}(d(i$, $j$ ) denotes distance between vertex $i$ and $j$; if vertex $i$ and $j$ are not connected, $\frac{1}{d(i, j)}$ is defined as $0 ; n$ is the number vertices in the network; in our case, $E\left(G^{\text {ideal }}\right)$ $=1)$; weigthed network connectance $=\frac{\sum_{i \neq j} w(i, j)}{n^{2}}(w(i, j)$ denoted the weight of the edge between vertex $i$ and j).

\section{Functional analysis}

We processed HMP Unified Metabolic Analysis Network (HUMAnN2) to determine the abundance of gene families [50]. MetaPhlAn2 and ChocoPhlAn pangenome database were used for functional profiling. Gene families determined by UniRef were mapped to Kyoto Encyclopedia of Genes and Genomes (KEGG) Orthogroups (KO and Gene Ontology (GO) database and were grouped into functional categories. The conditioned Mann-Whitney test estimated the $P$ values for the abundance change of the $\mathrm{KO} / \mathrm{GO}$ categories from control to CRC in each cohort and the MaxP method was used to determine the overall $\mathrm{f}$ for abundance change [51]. Spearman correlation was applied to estimate association strengths in determining the relationship between bacteria and $\mathrm{KO} / \mathrm{GO}$ categories.

\section{Additional files}

Additional file 1: Figure S1. (A) Shannon diversities of control and CRC samples in each cohort. (B) Combining the normalized Shannon diversity of all the control and CRC samples. The Shannon diversity was normalized to mean $=0$ and standard deviation $=1 .($ PDF $606 \mathrm{~kb})$

Additional file 2: Text Removing species with divergent abundance change directions. (DOCX $14 \mathrm{~kb}$ )

Additional file 3: Table S14. Significance of the abundance changes with rank sum test. (XLSX $12 \mathrm{~kb}$ )

Additional file 4: Table S4. Correlation between differentially abundant bacteria in early CRC. (XLSX $21 \mathrm{~kb})$

Additional file 5: Table S1. (DOCX $14 \mathrm{~kb})$

Additional file 6: Figure S5. Boxplot of the three species whose abundance significantly decreased between control, early-, and late-stage CRC $\left(P<0.05,{ }^{*} P<0.01,{ }^{* *} P<0.001,{ }^{* * *}\right)$. (PDF $\left.342 \mathrm{~kb}\right)$

Additional file 7: Figure S3. (A) Prediction performance of seven CRCenriched bacteria using a single model. (B) Prediction performance of the optimized the model of the individual cohort. (PDF $524 \mathrm{~kb}$ )

Additional file 8: Figure S12. (A) Prediction performance of the SVM model on the testing folds using 10-fold cross validation. (B) Prediction performance of the SVM model on the testing cohort using 'leave-onecohort-out' approach. (PDF $125 \mathrm{~kb}$ )

Additional file 9: Figure S4. (A) The precision and its corresponding recall index under different cut-off. The optimal F1 score was also provided. (B-C) With 271 controls and 30 cases, simulate probabilities with $A \cup C \approx 0.80$. Panel $B$ shows a simulated precision and recall graph.
Panel shows histogram of the optimal F1 scores with 500 simulations. (PDF $213 \mathrm{~kb}$ )

Additional file 10: Table S13. (XLSX $46 \mathrm{~kb}$ )

Additional file 11: Table S15. Markers selected by leave-one-cohortout. (XLSX $9 \mathrm{~kb})$

Additional file 12: Figure S6. (A) The table includes pfp of the seven CRC-enriched bacteria using all the four cohorts and three cohorts besides USA cohort. (B) Prediction performance of using seven CRCenriched bacteria to classify early-stage CRC from control. (PDF $900 \mathrm{~kb}$ )

Additional file 13: Figure S7. (A) Correlation between the 69 CRC differentially abundant bacteria on CRC samples. The left circle includes the CRC-depleted bacteria and the right includes the CRC-enriched bacteria. (B) The correlation between the 69 CRC differentially abundant bacteria in control. Node attributes are attached in Additional file 15: Table S2 and Additional file 28: Table S3. (PDF 334 kb)

Additional file 14: Figure S9. (A)-(B) Correlations between the 69 differentially abundant bacteria in early- and late-stage CRC. Node attributes are attached in Additional file 4: Table S4 and Additional file 24: Table S5. (C) Comparison between the correlation networks among CRC-enriched bacteria in early- and late-stage CRC. (PDF $927 \mathrm{~kb}$ )

Additional file 15: Table S2. Correlation between differentially abundant bacteria in Control. (XLSX $22 \mathrm{~kb}$ )

Additional file 16: Figure S8. Connectivity of the correlation network between the CRC-depleted bacteria in control samples. (PDF $367 \mathrm{~kb}$ )

Additional file 17: Table S6. Differentially Abundant GO categories. (XLSX $17 \mathrm{~kb}$ )

Additional file 18: Table S7. Differentially Abundant $K O$ categories. (XLSX $15 \mathrm{~kb}$ )

Additional file 19: Table S10. Correlation between bacteria correlatedCRC-enriched KO categories. (XLSX $22 \mathrm{~kb}$ )

Additional file 20: Figure S11. Correlation network between CRCdepleted bacteria and CRC-depleted GO/KO categories (correlations with $\rho>0.5$ were labeled in the figure). Node attributes are attached in Additional file 25: Table S11 and Additional file 26: Table S12. (PDF $3924 \mathrm{~kb}$ )

Additional file 21: Figure S10. Correlation network between CRCenriched bacteria and CRC-enriched GO categories. Node attributes are attached in Additional file 27: Table S9. (PDF $376 \mathrm{~kb}$ )

Additional file 22: Figure S13. Null distribution of the number of species left by removing the low abundant species, species missing in any cohort, and species with 'divergent abundance change directions'. (PDF $117 \mathrm{~kb}$ )

Additional file 23: Figure S2. Stratification of age in HK and FD cohorts. Conditioned Mann-Whitney $U$ test was performed conditioned on the age strata. (PDF $214 \mathrm{~kb}$ )

Additional file 24: Table S5. Correlation between differentially abundant bacteria in late CRC. (XLSX $21 \mathrm{~kb})$

Additional file 25: Table S9. Node attributes of the correlation network between CRC-enriched bacteria and GO categories. (XLSX $171 \mathrm{~kb}$ )

Additional file 26: Table S11. Correlation network between the CRCdepleted bacteria and KO categories. (XLSX $81 \mathrm{~kb}$ )

Additional file 27: Table S12. Correlation network between the CRC depleted bacteria and GO categories. (XLSX $29 \mathrm{~kb}$ )

Additional file 28: Table S3. Correlation between differentially abundant bacteria in CRC. (XLSX $19 \mathrm{~kb})$

Additional file 29: Table S8. Significant correlations between CRCenriched bacteria and KO categories. (XLSX $28 \mathrm{~kb}$ )

\section{Abbreviations}

AUC: Area under the receiver-operating characteristics curve; BMI: Body mass index; CRC: Colorectal cancer; FDR: False discovery rate; GO: Gene Ontology; HUMAnN2: HMP Unified Metabolic Analysis Network; KO: Kyoto Encyclopedia of Genes and Genomes (KEGG) Orthogroups; PC: Principal component; PCoA: Principal coordinates analysis; SVM: Support vector machine 


\section{Acknowledgements}

Not applicable

\section{Funding}

This project was supported by RGC-GRF Hong Kong $(766613,14106145$, 14111216), 135 program project China (2016YFC1303200), 973 Program China (2013CB531401), the National Key Technology R\&D Program (2014BAI09B05), Shenzhen Virtual University Park Support Scheme to CUHK Shenzhen Research Institute and CUHK Faculty of Medicine Grant on Microbiota Research.

\section{Availability of data and materials}

Full documentation of metadata, taxonomy read counts, and main data sets used during the current study are available on GitHub (https://github.com/ DAIZHENWEI/meta-analysis-microbiome).

\section{Authors' contributions}

ZD and OOC analyzed data and drafted the manuscript. GN and WKKW organized the data and drafted Figures. ZC, FKLC, KK, and JJYS commented the study and revised the manuscript. JY and SHW designed, supervised the study, and revised the paper. All authors read and approved the final manuscript.

\section{Ethics approval and consent to participate}

The study conformed to the ethical principles outlined by the Declaration of Helsinki and was approved by the Institutional Review Boards of the Chinese University of Hong Kong.

\section{Consent for publication}

Not applicable

\section{Competing interests}

The authors declare that they have no competing interest.

\section{Publisher's Note}

Springer Nature remains neutral with regard to jurisdictional claims in published maps and institutional affiliations.

\section{Author details}

${ }^{1}$ Institute of Digestive Disease and Department of Medicine and Therapeutics, State Key Laboratory of Digestive Disease, Li Ka Shing Institute of Health Sciences, The Chinese University of Hong Kong, Shatin, Hong Kong. ${ }^{2} \mathrm{CUHK}$ Shenzhen Research Institute, The Chinese University of Hong Kong, Shatin, Hong Kong. ${ }^{3}$ Department of Microbiology, Faculty of Medicine, The Chinese University of Hong Kong, Shatin, Hong Kong. ${ }^{4}$ Laboratory of Genomics and Molecular Biomedicine, Department of Biology, University of Copenhagen, Copenhagen, Denmark. ${ }^{5}$ Institute of Metagenomics BGI-Shenzhen, Shenzhen, China.

Received: 22 September 2017 Accepted: 19 March 2018 Published online: 11 April 2018

\section{References}

1. Ferlay J, Soerjomataram I, Dikshit R, Eser S, Mathers C, Rebelo M, Parkin DM, Forman D, Bray F. Cancer incidence and mortality worldwide: sources, methods and major patterns in GLOBOCAN 2012. Int J Cancer. 2015;136(5): E359-86.

2. Zhao LY, Zhang X, Zuo T, Yu J. The composition of colonic commensal Bacteria according to anatomical localization in colorectal Cancer. Engineering. 2017;3(1):90-7.

3. Kostic AD, Chun EY, Robertson L, Glickman JN, Gallini CA, Michaud M, Clancy TE, Chung DC, Lochhead P, Hold GL, et al. Fusobacterium nucleatum potentiates intestinal tumorigenesis and modulates the tumor-immune microenvironment. Cell Host Microbe. 2013;14(2):207-15.

4. Goodwin AC, Shields CED, Wu SG, Huso DL, Wu XQ, Murray-Stewart TR, Hacker-Prietz A, Rabizadeh S, Woster PM, Sears CL, et al. Polyamine catabolism contributes to enterotoxigenic Bacteroides fragilis-induced colon tumorigenesis. P Natl Acad Sci USA. 2011;108(37):15354-9.

5. Gao Z, Guo B, Gao R, Zhu Q, Qin H. Microbiota disbiosis is associated with colorectal cancer. Front Microbiol. 2015;6:20
6. Sobhani I, Tap J, Roudot-Thoraval F, Roperch JP, Letulle S, Langella P, Corthier G, Tran Van Nhieu J, Furet JP. Microbial dysbiosis in colorectal cancer (CRC) patients. PLoS One. 2011;6(1):e16393.

7. Ahn J, Sinha R, Pei ZH, Dominianni C, Wu J, Shi JX, Goedert JJ, Hayes RB, Yang LY. Human gut microbiome and risk for colorectal Cancer. Jnci J Natl Cancer I. 2013;105(24):1907-11.

8. Wang TT, Cai GX, Qiu YP, Fei N, Zhang MH, Pang XY, Jia W, Cai SJ, Zhao LP. Structural segregation of gut microbiota between colorectal cancer patients and healthy volunteers. Isme J. 2012;6(2):320-9.

9. Geng J, Fan $H$, Tang $X$, Zhai H, Zhang Z. Diversified pattern of the human colorectal cancer microbiome. Gut Pathog. 2013;5(1):2

10. Wong SH, Kwong TN, Chow T-C, Luk AK, Dai RZ, Nakatsu G, Lam TY, Zhang L, Wu JC, Chan FK. Quantitation of faecal fusobacterium improves faecal immunochemical test in detecting advanced colorectal neoplasia. Gut. 2016; gutjinl-2016-312766

11. Liang Q, Chiu J, Chen Y, Huang Y, Higashimori A, Fang J, Brim H, Ashktorab $\mathrm{H}, \mathrm{Ng}$ SC, Ng SSM. Fecal bacteria act as novel biomarkers for noninvasive diagnosis of colorectal cancer. Clin Cancer Res. 2017;23(8):2061-70.

12. Cohn LD, Becker BJ. How meta-analysis increases statistical power. Psychol Methods. 2003;8(3):243-53

13. Ross MG, Russ C, Costello M, Hollinger A, Lennon NJ, Hegarty R, Nusbaum $C$, Jaffe DB. Characterizing and measuring bias in sequence data. Genome Biol. 2013;14(5):R51.

14. Shah MS, DeSantis TZ, Weinmaier T, McMurdie PJ, Cope JL, Altrichter A, Yamal J-M, Hollister EB. Leveraging sequence-based faecal microbial community survey data to identify a composite biomarker for colorectal cancer. Gut. 2017; gutjnl-2016-313189

15. Langille MGI, Zaneveld J, Caporaso JG, McDonald D, Knights D, Reyes JA, Clemente JC, Burkepile DE, Thurber RLV, Knight R, et al. Predictive functional profiling of microbial communities using 165 rRNA marker gene sequences. Nat Biotechnol. 2013;31(9):814-21.

16. Yu J, Feng Q, Wong SH, Zhang D, Yi Liang Q, Qin Y, Tang L, Zhao H, Stenvang J, Li Y. Metagenomic analysis of faecal microbiome as a tool towards targeted non-invasive biomarkers for colorectal cancer. Gut. 2015; gutjnl-2015-309800

17. Segata N, Waldron L, Ballarini A, Narasimhan V, Jousson O, Huttenhower C. Metagenomic microbial community profiling using unique clade-specific marker genes. Nat Methods. 2012;9(8):811-4.

18. Fuller R. Probiotics in man and animals. J Appl Bacteriol. 1989;66(5):365-78.

19. Seki H, Shiohara M, Matsumura T, Miyagawa N, Tanaka M, Komiyama A Kurata S. Prevention of antibiotic-associated diarrhea in children by Clostridium butyricum MIYAIRI. Pediatr Int. 2003;45(1):86-90.

20. Burton JP, Wescombe PA, Moore CJ, Chilcott CN, Tagg JR. Safety assessment of the oral cavity probiotic Streptococcus salivarius K12. Appl Environ Microbiol. 2006;72(4):3050-3.

21. Horz HP, Meinelt A, Houben B, Conrads G. Distribution and persistence of probiotic Streptococcus salivarius K12 in the human oral cavity as determined by real-time quantitative polymerase chain reaction. Oral Microbiol Immunol. 2007:22(2):126-30.

22. Correa NBO, Peret LA, Penna FJ, Lima RMLS, Nicoli JR. A randomized formula controlled trial of Bifidobacterium lactis and Streptococcus thermophilus for prevention of antibiotic-associated diarrhea in infants. J Clin Gastroenterol. 2005:39(5):385-9.

23. Kim DH, Austin B. Innate immune responses in rainbow trout (Oncorhynchus mykiss, Walbaum) induced by probiotics. Fish Shellfish Immunol. 2006;21(5):513-24.

24. Roy D, Ward P, Vincent D, Mondou F. Molecular identification of potentially probiotic lactobacilli. Curr Microbiol. 2000;40(1):40-6.

25. Cortes C, Vapnik V. Support-vector networks. Mach Learn. 1995;20(3): 273-97.

26. Chong CW, Ahmad AF, Lim YAL, Teh CSJ, Yap IKS, Lee SC, Chin YT, Chua $\mathrm{KH}$. Effect of ethnicity and socioeconomic variation to the gut microbiota composition among pre-adolescent in Malaysia. Sci Rep U K. 2015:5:13338.

27. Prideaux L, Kang S, Wagner J, Buckley M, Mahar JE, De Cruz P, Wen ZH, Chen LP, Xia B, van Langenberg DR, et al. Impact of ethnicity, geography, and disease on the microbiota in health and inflammatory bowel disease. Inflamm Bowel Dis. 2013:19(13):2906-18.

28. Kwok L, Zhang J, Guo Z, Gesudu Q, Zheng Y, Qiao J, Huo D, Zhang H. Characterization of fecal microbiota across seven Chinese ethnic groups by quantitative polymerase chain reaction. PLoS One. 2014;9(4):e93631. 
29. Rhodes DR, Yu J, Shanker K, Deshpande N, Varambally R, Ghosh D, Barrette T, Pandey A, Chinnaiyan AM. Large-scale meta-analysis of cancer microarray data identifies common transcriptional profiles of neoplastic transformation and progression. Proc Natl Acad Sci U S A. 2004;101(25):9309-14.

30. Campain A, Yang YH. Comparison study of microarray meta-analysis methods. BMC Bioinformatics. 2010;11:408.

31. Feng Q, Liang SS, Jia HJ, Stadlmayr A, Tang LQ, Lan Z, Zhang DY, Xia HH, Xu $X Y$, Jie $Z Y$, et al. Gut microbiome development along the colorectal adenoma-carcinoma sequence. Nat Commun. 2015;6

32. Gur C, Ibrahim Y, Isaacson B, Yamin R, Abed J, Gamliel M, Enk J, Bar-On Y, Stanietsky-Kaynan N, Coppenhagen-Glazer S, et al. Binding of the Fap2 protein of fusobacterium nucleatum to human inhibitory receptor TIGIT protects tumors from immune cell attack. Immunity. 2015;42(2):344-55.

33. Weir TL, Manter DK, Sheflin AM, Barnett BA, Heuberger AL, Ryan EP. Stool microbiome and metabolome differences between colorectal cancer patients and healthy adults. PLoS One. 2013;8(8):e70803.

34. Whitmore SE, Lamont RJ. Oral bacteria and cancer. PLoS Pathog. 2014;10(3): e1003933.

35. Chen ZF, Ai LY, Wang JL, Ren LL, Yu YN, Xu J, Chen HY, Yu J, Li M, Qin WX et al. Probiotics Clostridium butyricum and Bacillus subtilis ameliorate intestinal tumorigenesis. Future Microbiol. 2015;10(9):1433-45.

36. Hsu RY, Chan CH, Spicer JD, Rousseau MC, Giannias B, Rousseau S, Ferri LE. LPS-induced TLR4 signaling in human colorectal cancer cells increases beta1 integrin-mediated cell adhesion and liver metastasis. Cancer Res. 2011;71(5):1989-98.

37. Rousseau MC, Hsu RY, Spicer JD, McDonald B, Chan CH, Perera RM, Giannias B, Chow SC, Rousseau S, Law S, et al. Lipopolysaccharide-induced toll-like receptor 4 signaling enhances the migratory ability of human esophageal cancer cells in a selectin-dependent manner. Surgery. 2013;154(1):69-77.

38. Coffelt SB, Wellenstein MD, de Visser KE. Neutrophils in cancer: neutral no more. Nat Rev Cancer. 2016;16(7):431-46.

39. Vogtmann E, Hua X, Zeller G, Sunagawa S, Voigt AY, Hercog R, Goedert JJ, Shi J, Bork P, Sinha R. Colorectal cancer and the human gut microbiome: reproducibility with whole-genome shotgun sequencing. PLoS One. 2016; 11(5):e0155362.

40. Zeller G, Tap J, Voigt AY, Sunagawa S, Kultima JR, Costea PI, Amiot A, Bohm J, Brunetti F, Habermann N, et al. Potential of fecal microbiota for earlystage detection of colorectal cancer. Mol Syst Biol. 2014;10:766.

41. Rosenbloom KR, Armstrong J, Barber GP, Casper J, Clawson H, Diekhans M, Dreszer TR, Fujita PA, Guruvadoo L, Haeussler M, et al. The UCSC genome browser database: 2015 update. Nucleic Acids Res. 2015;43(D1):D670-81.

42. Wood DE, Salzberg SL. Kraken: ultrafast metagenomic sequence classification using exact alignments. Genome Biol. 2014;15(3):R46.

43. Breitling $R$, Armengaud P, Amtmann A, Herzyk P. Rank products: a simple, yet powerful, new method to detect differentially regulated genes in replicated microarray experiments. FEBS Lett. 2004;573(1-3):83-92.

44. Hong F, Breitling R, McEntee CW, Wittner BS, Nemhauser JL, Chory J. RankProd: a bioconductor package for detecting differentially expressed genes in meta-analysis. Bioinformatics. 2006;22(22):2825-7.

45. Chang LC, Lin HM, Sibille E, Tseng GC. Meta-analysis methods for combining multiple expression profiles: comparisons, statistical characterization and an application guideline. BMC Bioinformatics. 2013;14:368.

46. Chen J, Li H. Variable selection for sparse Dirichlet-multinomial regression with an application to microbiome data analysis. Ann Appl Stat. 2013;7(1): 418-42.

47. Friedman J, Alm EJ. Inferring correlation networks from genomic survey data. PLoS Comput Biol. 2012;8(9):e1002687.

48. Hunter JE, Schmidt FL. Dichotomization of continuous-variables - the implications for metaanalysis. J Appl Psychol. 1990:75(3):334-49.

49. Hedges LV, Vevea JL. Fixed- and random-effects models in meta-analysis. Psychol Methods. 1998:3(4):486-504

50. Abubucker S, Segata N, Goll J, Schubert AM, Izard J, Cantarel BL, RodriguezMueller B, Zucker J, Thiagarajan M, Henrissat B. Metabolic reconstruction for metagenomic data and its application to the human microbiome. PLoS Comput Biol. 2012;8(6):e1002358.

51. Li J, Tseng GC. An adaptively weighted statistic for detecting differential gene expression when combining multiple transcriptomic studies. Ann Appl Stat. 2011:5(2A):994-1019.

\section{Submit your next manuscript to BioMed Central and we will help you at every step:}

- We accept pre-submission inquiries

- Our selector tool helps you to find the most relevant journal

- We provide round the clock customer support

- Convenient online submission

- Thorough peer review

- Inclusion in PubMed and all major indexing services

- Maximum visibility for your research

Submit your manuscript at www.biomedcentral.com/submit
Biomed Central 